\title{
Group Weighted Matchings in Bipartite Graphs
}

\author{
R. AHARONI, R. MESHULAM*AND B. WAJNRYB \\ Department of Mathematics, Technion, Haifa 32000, Israel \\ Received May 10, 1992; Revised April 5, 1994
}

\begin{abstract}
Let $G$ be a bipartite graph with bicoloration $\{A, B\},|A|=|B|$, and let $w: E(G) \rightarrow \mathbf{K}$ where $\mathbf{K}$ is a finite abelian group with $k$ elements. For a subset $S \subset E(G)$ let $w(S)=\prod_{\epsilon \in S} w(e)$. A perfect matching $M \subset E(G)$ is a $w$-matching if $w(M)=1$.

A characterization is given for all $w$ 's for which every perfect matching is a $w$-matching.

It is shown that if $G=K_{k+1, k+1}$ then either $G$ has no $w$-matchings or it has at least $2 w$-matchings

If $\mathbf{K}$ is the group of order 2 and $\operatorname{deg}(a) \geq d$ for all $a \in A$, then either $G$ has no $w$-matchings, or $G$ has at least $(d-1)$ ! w-matchings.
\end{abstract}

Keywords: bipartite matching, Abelian group

\section{Introduction}

Let $G$ be a bipartite graph with bicoloration $\{A, B\},|A|=|B|=n$. Let $E(G) \subset A \times B$ denote the edge set of $G, e(G)=|E(G)|$.

Let $\mathbf{K}$ be a (multiplicative) finite abelian group $|\mathbf{K}|=k$, and let $w: E(G) \rightarrow \mathbf{K}$ be a weight assignment on the edges of $G$. For a subset $S \subset E(G)$ let $w(S)=\prod_{e \in S} w(e)$.

A perfect matching $M$ of $G$ is a $w$-matching if $w(M)=1$. We shall consider several problems concerning $w$-matchings.

Let $F(G)=\mathbf{K}^{E(G)}$ denote all mappings $w: E(G) \rightarrow \mathbf{K}$. and let $M(G)$ denote all $w \in F(G)$ which satisfy $w(M)=1$ for all perfect matchings $M$ of $G$.

Aharoni, Manber and Wajnryb [1] obtained a concise description of $M(G)$ when $\mathbf{K}=\mathbf{C}_{2}$ is the group of order 2 . Here we give a new proof and an extension to arbitrary abelian groups.

One simple way of obtaining elements of $M(G)$ is the following: Choose $\alpha: A \rightarrow$ $\mathbf{K}, \beta: B \rightarrow \mathbf{K}$ which satisfy $\prod_{a \in A} \alpha(a) \prod_{b \in B} \beta(b)=1$, and define $w: E(G) \rightarrow \mathbf{K}$ by $w(a, b)=\alpha(a) \beta(b)$. Clearly $w \in M(G)$.

Denote by $U(G) \subset M(G)$ the set of all $w$ 's obtained this way.

Theorem 1.1 If every edge in $G$ is contained in a perfect matching then $U(G)=M(G)$.

The case $\mathbf{K}=\mathbf{C}_{2}$ of Theorem 1.1 was proved by Aharoni, Manber and Wajnryb [1].

Next we consider $w$-matchings in complete bipartite graphs.

Let $K_{k+1, k+1}$ denote the complete bipartite graph on $\{A, B\},|A|=|B|=k+1$, and let $w: E\left(K_{k+1, k+1}\right) \rightarrow \mathbf{K}$.

"Research supported by a Technion V.P.R. Grant No. 100-854. 
Theorem 1.2 If $K_{k+1, k+1}$ has a w-matching, then it has at least two $w$-matchings.

Finally we consider the number of $w$-matchings in bipartite graphs.

M. Hall (see exercise 7.15 in [4]) proved that if $G$ has a perfect matching and if $\operatorname{deg}(a) \geq d$ for all $a \in A$, Then $G$ has at least $d$ ! perfect matchings.

Here we show

Theorem 1.3 Let $w: E(G) \rightarrow \mathbf{C}_{2}$. If $G$ has a $w$-matching and $\operatorname{deg}(a) \geq d$ for all $a \in A$, then $G$ has at least $(d-1)$ ! w-matchings.

Theorem 1.1 is proved in section 2 . In section 3 we apply the group algebra of $\mathbf{K}$ to $w$-matchings in complete bipartite graphs. In section 4 we prove a result on $\mathbf{C}_{2}$-weighted digraphs which implies Theorem 1.3. A special case of Theorem 1.3 is then applied to a problem of Rinnot on random matrices. We conclude in section 5 with a conjecture which extends the results of sections 3 and 4 .

\section{Proof of Theorem 1.1}

We may clearly assume that $G$ is an elementary bipartite graph, i.e. $G$ is connected and every edge of $G$ is contained in a perfect matching.

By a result of Hetyei (exercise 7.7 in [4]) $G$ satisfies

$$
|\Gamma(X)|>|X| \text { whenever } \emptyset \neq X \subseteq A \text { or } \emptyset \neq X \subseteq B
$$

where $\Gamma(X)$ denotes the neighbors of $X$.

Note that $U(G) \subset M(G) \subset F(G)$ are abelian groups with respect to pointwise multiplication: $w_{1} w_{2}(e)=w_{1}(e) w_{2}(e)$.

We first prove a lower bound on $|U(G)|$.

Claim 2.2. $|U(G)| \geq k^{2 n-2}$.

Proof: Let $A=\left\{a_{1}, \ldots, a_{n}\right\}, B=\left\{b_{1}, \ldots, b_{n}\right\}$. Denote by $K^{m}$ the direct product $\mathbf{K} \times \cdots \times \mathbf{K}(m$ times $)$. Define a homomorphism $\Phi: \mathbf{K}^{n-1} \times \mathbf{K}^{n-1} \rightarrow U(G)$ as follows: Let $u=\left(u_{1}, \ldots, u_{n-1}\right), v=\left(v_{1}, \ldots, v_{n-1}\right) \in \mathbf{K}^{n-1}$, and set $u_{n}=\prod_{i=1}^{n-1} u_{i}^{-1} v_{i}^{-1}, v_{n}=1$.

Define $\Phi(u, v) \in M(G)$ by $\Phi(u, v)\left(a_{i}, b_{j}\right)=u_{i} v_{j}$ for $\left(a_{i}, b_{j}\right) \in E(G)$.

We show that $\Phi$ is 1-1. Suppose to the contrary that $(1,1) \neq(u, v) \in \operatorname{ker} \Phi \subset \mathbf{K}^{n-1} \times$ $\mathbf{K}^{n-1}$. Let $X=\left\{a_{i}: u_{i} \neq 1\right\}, Y=\left\{b_{j}: v_{j} \neq 1\right\}$. If $|X| \leq|Y|$ then since $|Y| \leq n-1$ it follows from 2.1 that $|\Gamma(Y)|>|X|$. Therefore there exists an edge $\left(a_{i}, b_{j}\right) \in E(G)$ such that $a_{i} \notin X$ and $b_{j} \in Y$. Thus $1=\Phi(u, v)\left(a_{i}, b_{j}\right)=u_{i} v_{j}=v_{j}$, a contradiction. The case $|X|>|Y|$ is similiar. Therefore $\Phi$ is $1-1$ and the Claim follows.

Denote by $\widehat{H}$ the character group of a finite abelian group $H$. For a subgroup $\Lambda \subset \widehat{H}$ let $\Lambda^{\perp}=\{h \in H: \chi(h)=1$ for all $\chi \in \Lambda\} . \Lambda^{\perp}$ is a subgroup of $H$ and $|\Lambda|\left|\Lambda^{\perp}\right|=|H|$.

For each $\chi \in \widehat{\mathbf{K}}$ and a perfect matching $M$ of $G$, let $c(M, \chi) \in \widehat{F(G)}$ be defined by $c(M, \chi)(w)=\chi(w(M))$. Let $P(G) \subset \widehat{F(G)}$ be the subgroup generated by all the $c(M, \chi)$ 's. Clearly $P(G)^{\perp}=M(G)$. We now prove a lower bound on $|P(G)|$. 
Claim 2.3. $|P(G)| \geq k^{e(G)-2(n-1)}$.

Proof: We argue by induction on $e(G)$. If $e(G)=1$ then $n=1$ and $P(G) \cong \widehat{\mathbf{K}}$. Suppose $e(G)>1$. By a theorem of Hetyei on the structure of elementary bipartite graphs (exercise 7.8 in [4]) $G$ decomposes as $G=G^{\prime} \cup C$, where $G^{\prime}$ is again elementary, and $C$ is an odd path joining $x \in V\left(G^{\prime}\right) \cap A$ and $y \in V\left(G^{\prime}\right) \cap B$ such that $V(C) \cap V\left(G^{\prime}\right)=\{x, y\}$.

To simplify notation assume that for some $1 \leq m \leq n$.

$V\left(G^{\prime}\right)=\left\{a_{1}, \ldots, a_{m}\right\} \cup\left\{b_{1}, \ldots, b_{m}\right\}, V(C)=\left\{a_{m}, \ldots, a_{n}\right\} \cup\left\{b_{m}, \ldots, b_{n}\right\}$ and $E(C)=$ $\left\{\left(a_{i}, b_{i-1}\right)\right\}_{i=m+1}^{n} \cup\left\{\left(a_{i}, b_{i}\right)\right\}_{i=m+1}^{n} \cup\left\{\left(a_{m}, b_{n}\right)\right\}$

We also choose a (fixed) perfect matching $\bar{M}$ of $G$ which contains the edge $\left(a_{m}, b_{n}\right)$.

Every perfect matching $M^{\prime}$ of $G^{\prime}$ can be extended to a perfect matching $\epsilon\left(M^{\prime}\right)=M$ by $M=M^{\prime} \cup\left\{\left(a_{i}, b_{i}\right)\right\}_{i=m+1}^{n}$.

Define $h: P\left(G^{\prime}\right) \times \widehat{\mathbf{K}} \rightarrow P(G)$ as follows: Let $\varphi=\prod_{i=1}^{t} c\left(M_{i}^{\prime}, \chi_{i}\right) \in P\left(G^{\prime}\right)$ where $\chi_{i} \in$ $\widehat{K}$ and the $M_{i}^{\prime}$ 's are perfect matchings of $G^{\prime}$. Define $h(\varphi, \chi)=\prod_{i=1}^{t} c\left(\epsilon\left(M_{i}^{\prime}\right), \chi_{i}\right) c(\bar{M}, \chi)$.

We check that $h$ is 1-1. Suppose $(\psi, \eta)=\left(\prod_{j=1}^{t} c\left(N_{j}^{\prime}, \eta_{j}\right), \eta\right) \in P\left(G^{\prime}\right) \times \widehat{\mathbf{K}}$ where $\eta_{j} \in \widehat{\mathbf{K}}$ and the $N_{j}^{\prime}$ 's are perfect matchings of $G^{\prime}$.

If $\chi \neq \eta$ then $\chi(z) \neq \eta(z)$ for some $z \in K$. Define $w \in F(G)$ by $w(e)=z$ if $e=\left(a_{m}, b_{n}\right)$ and $w(e)=1$ otherwise. Clearly $h(\varphi, \chi)(w)=\chi(z) \neq \eta(z)=h(\psi, \eta)(w)$. If on the other hand $\chi=\eta$, then $\varphi \neq \psi$ and so $\varphi\left(w^{\prime}\right) \neq \psi\left(w^{\prime}\right)$ for some $w^{\prime} \in F\left(G^{\prime}\right)$. Defining $w \in F(G)$ by $w(e)=w^{\prime}(e)$ for $e \in E\left(G^{\prime}\right)$ and $w(e)=1$ otherwise, we obtain $h(\varphi, \chi)(w)=\varphi\left(w^{\prime}\right) \chi(\omega(\bar{M})) \neq \psi\left(w^{\prime}\right) \chi(\omega(\bar{M}))=h(\psi, \eta)(w)$.

The injectivity of $h$ together with the induction hypothesis imply:

$$
|P(G)| \geq\left|P\left(G^{\prime}\right)\right| \cdot|\widehat{\mathbf{K}}| \geq k^{e\left(G^{\prime}\right)-2(m-1)+1}=k^{e(G)-2(n-1)}
$$

Claims 2.2 and 2.3 imply

$$
k^{2 n-2} \leq|U(G)| \leq|M(G)|=\left|P(G)^{\perp}\right|=(\widehat{F(G)}: P(G))=k^{e(G)} /|P(G)| \leq k^{2 n-2}
$$

Therefore $U(G)=M(G)$.

\section{$3 w$-matchings in complete bipartite graphs}

Let $M_{m}(S)$ denote all $m \times m$ matrices with entries in $S$.

For $Q=\left(q_{i j}\right) \in M_{m}(\mathbf{K})$ and a permutation $\sigma \in S_{m}$, let $Q(\sigma)=\prod_{i=1}^{m} a_{i \sigma(i)}$. For $x \in \mathbf{K}$ let $S(Q, x)=\left\{\sigma \in S_{m}: Q(\sigma)=x\right\}$.

Let $t=t(\mathbf{K})$ denote the minimal $t$ such that for any $Q \in M_{t}(\mathbf{K})$, either $S(Q, 1)=\emptyset$ or $|S(Q, 1)| \geq 2$.

A mapping $w: E\left(K_{m, m}\right) \rightarrow \mathbf{K}$ naturally corresponds to a matrix $Q \in M_{m}(\mathbf{K})$. We prove the following matrix version of Theorem 1.2.

Theorem $3.1 \quad t(\mathbf{K}) \leq k+1$.

Proof: Let $Q=\left(q_{i j}\right) \in M_{k+1}(\mathbf{K})$. Denote by $\mathbf{C}[\mathbf{K}]$ the complex group algebra of $\mathbf{K}$ and let $\widehat{\mathbf{K}}=\left\{\chi_{1}, \ldots, \chi_{k}\right\}$. 
Define $\left(\lambda_{i j}\right) \in M_{k+1}\left(\mathbf{C}^{*}\right)$ by $\lambda_{1 j}=1$ and $\lambda_{i j}=\chi_{i-1}\left(q_{1 j} q_{i j}^{-1}\right)$ for all $2 \leq i \leq k+1$, $1 \leq j \leq k+1$. Let $R=\left(r_{i j}\right) \in M_{k+1}(\mathbf{C}[\mathbf{K}])$ be defined by $r_{i j}=\lambda_{i j} q_{i j}$. Note that $\operatorname{det} R \in \mathbf{C}[\mathbf{K}]$.

Claim 3.2. $\operatorname{det} R=0$.

Proof: Let $1 \leq l \leq k$ and consider the matrix $\chi_{l}(R)=\left(\chi_{l}\left(r_{i j}\right)\right) \in M_{k+1}(\mathbf{C})$.

Clearly $\chi_{l}\left(r_{1 j}\right)=\chi_{l}\left(r_{l+1, j}\right)$ for all $1 \leq j \leq k+1$, therefore $\chi_{l}(R)$ is singular and $\chi_{l}(\operatorname{det} R)=\operatorname{det}\left(\chi_{l}(R)\right)=0$. Since this holds for all $1 \leq l \leq k$ it follows that $\operatorname{det} R=0$.

Therefore

$$
0=\operatorname{det} R=\sum_{x \in \mathbf{K}}\left(\sum_{\sigma \in S(Q, x)} S g(\sigma) \prod_{i=1}^{k+1} \lambda_{i \sigma(i)}\right) x
$$

So that for each $x \in \mathbf{K}$ either $S(Q, x)=\emptyset$ or $|S(Q, x)| \geq 2$.

A lower bound on $t(\mathbf{K})$ may be obtained as follows: Let $s=s(\mathbf{K})$ denote the maximal $s$ for which there exists a sequence $x_{1}, \ldots, x_{s} \in \mathbf{K}$ such that $\prod_{i \in I} x_{i} \neq 1$ for all $\emptyset \neq I \subset$ $\{1, \ldots, s\}$.

Define $Q=\left(q_{i j}\right) \in M_{s+1}(\mathbf{K})$ by $q_{i j}=1$ if $i=j$ or $i=s+1$, and $q_{i j}=x_{i}$ otherwise. Clearly $S(Q, 1)$ contains only the identity permutation, so $t(\mathbf{K}) \geq s(\mathbf{K})+2$. Note that for the cyclic group $\mathbf{K}=\mathbf{C}_{k}$ this lower bound is tight by Theorem 3.1.

$s(\mathbf{K})$ was studied by a number of authors ([6], [3], [2], [5]). We shall need the following result of Olson. Let $\mathbf{Z}_{p}[\mathbf{K}]$ denote the group algebra of $\mathbf{K}$ with coefficients in $\mathbf{Z}_{p}$.

Theorem (Olson [6]) Let $\mathbf{K}$ be an abelian p-group $\mathbf{K}=\mathbf{C}_{p^{e_{1}}} \times \cdots \times \mathbf{C}_{p^{e_{1}}}$. Then $s=$ $s(\mathbf{K})=\sum_{i=1}^{l}\left(p^{e_{i}}-1\right)$ and for every $x_{1}, \ldots, x_{i+1} \in \mathbf{K}, \prod_{i=1}^{s+1}\left(x_{i}-1\right)=0$ in $\mathbf{Z}_{p}[\mathbf{K}]$.

We now show

Theorem 3.3 If $\mathbf{K}$ is an abelian p-group, then $t(\mathbf{K})=s(\mathbf{K})+2$.

Proof: Let $s=s(\mathbf{K})$ and let $Q=\left(q_{i j}\right) \in M_{s+2}(\mathbf{K})$. As in Theorem 3.1 it suffices to show that det $Q=0$ in $\mathbf{Z}_{p}[\mathbf{K}]$. Multiplying rows and columns by appropriate elements of $\mathbf{K}$ we may assume that $q_{1 i}=q_{i 1}=1$ for all $1 \leq i \leq s+2$. Subtracting the first row from the others, we obtain:

$$
\operatorname{det} Q=\sum_{\sigma} S g(\sigma) \prod_{i=2}^{i+2}\left(q_{i \sigma(i)}-1\right)
$$

where $\sigma$ ranges over all permutations of $2, \ldots, s+2$. By Olson's Theorem all products on the right vanish and so det $Q=0$.

In section 4 we shall need a version of Theorem 3.1 for directed graphs. Let $\vec{K}_{k+1}$ denote the complete directed graph on $V=\{1, \ldots, k+1\}, E\left(\vec{K}_{k+1}\right)=\{(i, j): 1 \leq i \neq j \leq k+1\}$. For $w: E\left(\vec{K}_{k+1}\right) \rightarrow \mathbf{K}$ and $S \subset E\left(\vec{K}_{k+1}\right)$ let $w(S)=\prod_{e \in S} w(e)$. 
Corollary 3.4 For any $w: E\left(\vec{K}_{k+1}\right) \rightarrow \mathbf{K}$ there exist vertex disjoint directed cycles $C_{1}, \ldots, C_{l}$ in $\vec{K}_{k+1}$ such that $\prod_{i=1}^{l} w\left(C_{i}\right)=1$.

Proof: Define $Q=\left(q_{i j}\right) \in M_{k+1}(\mathbf{K})$ by $q_{i i}=1$ and $q_{i j}=w(i, j)$ for $1 \leq i \neq j \leq k+1$. Since the identity permutation belong to $S(Q, 1)$, it follows from Theorem 3.1 that there exists a $1 \neq \sigma \in S(Q, 1)$.

$V_{0}=\{i: \sigma(i) \neq i\}$ clearly decomposes into vertex disjoint directed cycles $C_{1}, \ldots, C_{l}$ such that $\prod_{i=1}^{l} w\left(C_{i}\right)=\prod_{j=1}^{n} q_{j \sigma(j)}=1$.

\section{On the number of $w$-matchings}

Let $D=(V, E)$ be a directed graph, possibly with loops but with no multiple edges in the same direction.

The proof of Theorem 1.3 depends on the following result which combines an idea of Thomassen [8] with Corollary 3.4 .

Proposition 4.1 Let $D=(V, E)$ be a digraph (as above), and let $w: E \rightarrow \mathbf{C}_{2}$. If $\operatorname{deg}^{+}(v)=2$ for all $v \in V$, then there exist vertex disjoint directed cycles $C_{1}, \ldots, C_{l}$ such that $\prod_{i=1}^{l} w\left(C_{i}\right)=1$.

Proof: Let $D$ be a minimal counterexample. If $C_{1}, C_{2}$ are two vertex disjoint directed cycles then either $w\left(C_{i}\right)=1$ for some $i$, or $w\left(C_{1}\right) w\left(C_{2}\right)=1$. It follows that any two dicycles intersect. If $D$ has a loop $C_{1}=(v, v)$ then $D-v$ has a directed cycle $C_{2}$, thus $D$ is loopless.

Suppose there is an edge $(x, y) \in E$ such that for no $v \in V$ both $(v, x)$ and $(v, y)$ are edges. We form a new digraph $D^{\prime}=\left(V^{\prime}, E^{\prime}\right)$ on $V^{\prime}=V-x$ by deleting $x$ and all edges incident with it, and replacing each edge of the form $(v, x) \in E$ by a new edge $(v, y) \in E^{\prime}$. Note that $\operatorname{deg}^{+}\left(v^{\prime}\right)=2$ for all $v^{\prime} \in V^{\prime}$. Define $w^{\prime}: E^{\prime} \rightarrow \mathbf{C}_{2}$ by $w^{\prime}\left(e^{\prime}\right)=w\left(e^{\prime}\right)$ for $e^{\prime} \in E$, and $w^{\prime}(v, y)=w(v, x) w(x, y)$ if $(v, x) \in E$

With each directed cycle $C^{\prime}$ in $D^{\prime}$ we associate a directed cycle $C$ in $D$. If $C^{\prime}$ contains a new edge $(v, y) \in E^{\prime}$ (where $(v, x) \in E$ ), let

$C=C^{\prime}-(v, y)+(v, x)+(x, y)$. Otherwise $C=C^{\prime}$. Clearly $w(C)=w^{\prime}\left(C^{\prime}\right)$ and $V\left(C_{1}^{\prime}\right) \bigcap V\left(C_{2}^{\prime}\right)=\emptyset$ implies $V\left(C_{1}\right) \cap V\left(C_{2}\right)=\emptyset$. Therefore if $D^{\prime}$ satisfies the conclusions of the Theorem, so does $D$-in contradiction with the minimality assumption.

Therefore for every $(x, y) \in E$ there exists a vertex $z \neq x, y$ such that $(z, x),(z, y) \in E$. It follows that each $v \in V$ is dominated by a directed cycle, and in particular $\operatorname{deg}^{-}(v) \geq 2$. Since $\operatorname{deg}^{+}(v)=2$ for all $v$, it follows that there exists a $v$ such that $\operatorname{deg}^{-}(v)=2$. Thus there is a cycle $C_{1}=\{(x, y),(y, x)\}$ such that $(x, v),(y, v) \in E$.

Let $C_{2}$ be a cycle which dominates $x$. Clearly $y \in V\left(C_{2}\right)$ for otherwise $C_{1}$ and $C_{2}$ are vertex disjoint. Therefore $v \in V\left(C_{2}\right)$ too, and so $(v, x) \in E$. Similarly we conclude that $(v, y) \in E$.

Therefore the complete directed graph on $\{x, y, v\}$ is contained in $D$, in contradiction with Corollary 3.4 (for the group $\mathbf{K}=\mathbf{C}_{2}$ ).

Returning to the number of $w$-matchings, let $G$ be a bipartite graph on $\{A, B\},|A|=$ $|B|=n$ and $w: E(G) \rightarrow \mathbf{C}_{2}$. For $a \in A$ let $U_{G}(a, w)$ denote the set of all edges incident with $a$ which participate in a $w$-matching of $G,\left|U_{G}(a, w)\right|=u_{G}(a, w)$. 
The following result clearly implies Theorem 1.3 by induction on $d$.

Theorem 4.2 If $G$ has a w-matching then there exists an $a \in A$ such that $u_{G}(a, w) \geq$ $\operatorname{deg}_{G}(a)-1$.

Proof: We argue by induction on $e(G)$. Let $\delta(G)=\min \left\{\operatorname{deg}_{G}(a): a \in A\right\}$. The assertion is clear if $\delta(G) \leq 2$, so we assume $\delta(G) \geq 3$.

Suppose there exists an $a \in A$ with $\operatorname{deg}_{G}(a) \geq 4$ and distinguish two cases:

a) $U_{G}(a, w)=\{e\}$. Choose $e^{\prime} \neq e$ incident with $a$ and let $G^{\prime}=G-e^{\prime}$. By induction there exists an $a^{\prime} \in A$ such that $u_{G^{\prime}}\left(a^{\prime}, w\right) \geq \operatorname{deg}_{G^{\prime}}\left(a^{\prime}\right)-1$. Since $u_{G}(a, w)=1$ and $\operatorname{deg}_{G^{\prime}}(a) \geq 3$, it follows that $a^{\prime} \neq a$ and so $u_{G}\left(a^{\prime}, w\right)=u_{G^{\prime}}\left(a^{\prime}, w\right) \geq \operatorname{deg}_{G}\left(a^{\prime}\right)-1$.

b) $U_{G}(a, w) \supset\left\{e, e^{\prime}\right\}$. Again let $G^{\prime}=G-e^{\prime}$ and choose by induction an $a^{\prime} \in A$ such that $u_{G^{\prime}}\left(a^{\prime}, w\right) \geq \operatorname{deg}_{G^{\prime}}\left(a^{\prime}\right)-1$. If $a^{\prime} \neq a$ we are done as before. Otherwise $a^{\prime}=a$ and so $U_{G}(a, w)=U_{G^{\prime}}(a, w) \bigcup\left\{e^{\prime}\right\}$. Therefore

$$
u_{G}(a, w)=u_{G^{\prime}}(a, w)+1 \geq\left(\operatorname{deg}_{G^{\prime}}(a)-1\right)+1=\operatorname{deg}_{G}(a)-1 .
$$

We thus remain with the case $\operatorname{deg}(a)=3$ for all $a \in A$.

Let $M=\left\{\left(a_{1}, b_{1}\right), \ldots,\left(a_{n}, b_{n}\right)\right\}$ be a $w$-matching of $G$. With no loss of generality we may assume that $w\left(a_{i}, b_{i}\right)=1$ for all $i$. Construct a directed graph $D$ on $\{1, \ldots, n\}$ by $(i, j) \in E(D)$ iff $i \neq j$ and $\left(a_{i}, b_{j}\right) \in E(G)$, and let $\varphi: E(D) \rightarrow \mathbf{C}_{2}$ be defined by $\varphi(i, j)=w\left(a_{i}, b_{j}\right)$. Since $\operatorname{deg}^{+}(v)=2$ for all $v \in V(D)$, it follows from Proposition 4.1 that there exist vertex disjoint cycles $C_{1}, \ldots, C_{l}$ such that $\prod_{i=1}^{l} w\left(C_{i}\right)=1$. Let $V_{0}=\bigcup_{i=1}^{l} V\left(C_{i}\right)$ and define a permutation $\sigma$ on $V_{0}$ by $\sigma\left(v_{1}\right)=v_{2}$ if $\left(v_{1}, v_{2}\right) \in \bigcup_{i=1}^{l} E\left(C_{i}\right)$. Consider the perfect matching

$$
M^{\prime}=\left\{\left(a_{i}, b_{i}\right): i \notin V_{0}\right\} \bigcup\left\{\left(a_{i}, b_{\sigma(i)}\right): i \in V_{0}\right\} .
$$

Clearly $M^{\prime} \neq M$ and $w\left(M^{\prime}\right)=\prod_{i=1}^{l} \varphi\left(C_{i}\right)=1$.

Applying Theorem 1.3 to the complete bipartite graph $K_{n, n}$ we obtain

Corollary 4.3 Let $Q=\left(q_{i j}\right) \in M_{n}\left(\mathbf{C}_{2}\right)$. Then either $S(Q, 1)=\emptyset$ or $|S(Q, 1)| \geq$ $(n-1)$ !.

We conclude this section with an application of Corollary 4.3.

Let $X=\left(X_{i j}\right)$ be an $n \times n$ matrix of independent random variables $X_{i j}$ such that $\operatorname{Pr}\left(X_{i j}=\right.$ $1)=\operatorname{Pr}\left(X_{i j}=-1\right)=1 / 2$. For $\sigma \in S_{n}$, define a random variable $X(\sigma)=\prod_{i=1}^{n} X_{i \sigma(i)}$ and let id be the identity permutation in $S_{n}$.

Denote by $f(n)$ the maximal cardinality of a family of permutations $S \subset S_{n}$ such that $X$ (id) is independent of $\{X(\sigma): \sigma \in S\}$. Y. Rinnot [7] noted that $S=\left\{\sigma \in S_{n}: \sigma(1) \neq 1\right\}$ satisfies this independence condition and thus $f(n) \geq|S|=n !-(n-1)$ !. Here we show that Rinnot's construction is optimal:

Theorem 4.4 If $X(\mathrm{id})$ is independent of $\{X(\sigma): \sigma \in S\}$, then $|S| \leq n !-(n-1) !$.

Proof: The events $A=\{X(\sigma)=-1$ for all $\sigma \in S\}$ and $B=\{X$ (id) $=1\}$ are clearly independent and both have positive probability, therefore $\operatorname{Pr}(A \cap B)=\operatorname{Pr}(A) \operatorname{Pr}(B)>0$. 
Hence there exists a matrix $Q \in M_{n}( \pm 1)$ such that $Q(\sigma)=-1$ for all $\sigma \in S$ and $Q($ id) $=1$. Therefore $S(Q, 1) \cap S=\emptyset$ and $S(Q, 1) \neq \emptyset$, so by Corollary 4.3

$$
|S| \leq n !-|S(Q, 1)| \leq n !-(n-1) ! .
$$

\section{Concluding remarks}

Our results seem to suggest the following extension of Theorem 1.3.

Conjecture 5.1 Let $G$ be a bipartite graph on $\{A, B\},|A|=|B|$, and let $w: E(G) \rightarrow \mathbf{K}$. If $G$ has $a$ w-matching and $\operatorname{deg}(a) \geq d$ for all $a \in A$, then $G$ has at least $(d-s(\mathbf{K}))$ ! $w$-matchings.

The proof of Theorem 4.2 may be modified to show that Conjecture 5.1 is equivalent to

Conjecture 5.2 Let $D=(V, E)$ be a simple digraph, and let $w: E \rightarrow \mathbf{K}$. If $\operatorname{deg}^{+}(v)=$ $s(\mathbf{K})+1$ for all $v \in V$, Then there exist vertex disjoint directed cycles $C_{1}, \ldots, C_{l}$ such that $\prod_{i=1}^{l} w\left(C_{i}\right)=1$.

\section{Remarks}

a) The lower bound $t(\mathbf{K}) \geq s(\mathbf{K})+2$ shows that the conjectures do not hold if $s(\mathbf{K})$ is replaced by a smaller constant.

b) Both conjectures hold when $s(\mathbf{K})$ is replaced by another (much larger) constant $c(\mathbf{K})$.

Added on June 1, 1993: J. Kahn and R. Meshulam proved that both conjectures hold when $s(\mathbf{K})$ is replaced by $|\mathbf{K}|-1$. In particular the conjectures are valid for cyclic $\mathbf{K}$. Details will appear elsewhere.

\section{References}

1. R. Aharoni, R. Manber, and B. Wajnryb, "Special parity of perfect matchings in bipartite graphs," Discrete Math. 79 (1989/1990), 221-228.

2. R. C. Baker and W. M. Schmidt, "Diophantine problems in variables restricted to the values 0 and $1, " J$. Number Theory 12 (1980), 460-486.

3. P. Van Emde Boas and D. Kruyswijk, "A combinatorial problem on finite abelian groups III," Z.W. 1969-008 (Math. Centrum, Amsterdam).

4. L. Lovász, Combinatorial problems and exercises, North-Holland, New York, 1979.

5. R. Meshulam, "An uncertainty inequality and zero subsums," Discrete Math. 84 (1990), 197-200

6. J. E. Olson, "A combinatorial problem on finite abelian groups I," J. Number Theory 1 (1969), 8-10.

7. Y. Rinnot, Private communication, November 1991.

8. C. Thomassen, "Disjoint cycles in digraphs," Combinatorica 3 (1983), 393-396. 